\title{
Asymmetric Total Synthesis of Nigerone
}

Evan S. DiVirgilio, Elizabeth C. Dugan, Carol A. Mulrooney, and Marisa C. Kozlowski* Department of Chemistry, Roy and Diana Vagelos Laboratories, University of Pennsylvania, Philadelphia, Pennsylvania 19104-6323 marisa@sas.upenn.edu

General Considerations:

Synthetic Procedures:

NMR Spectra:

References:
S2

S3-S8

S9-S18

S19 
General Considerations. Unless otherwise noted, all non-aqueous reactions were carried out under an atmosphere of dry $\mathrm{N}_{2}$ in dried glassware. When necessary, solvents and reagents were dried prior to use. Toluene and $\mathrm{CH}_{2} \mathrm{Cl}_{2}$ were de-oxygenated by purging with $\mathrm{N}_{2}$ and then dried by passing through activated alumina. THF was distilled from sodium benzophenone ketyl. $\mathrm{CH}_{3} \mathrm{CN}$, TMEDA, and hexanes were distilled from $\mathrm{CaH}_{2}$. Benzene was distilled from sodium. Solvents for the preparation of the catalyst complexes and for the oxidative coupling reactions were usually used without purification although acid-free halogenated solvents are required (if necessary, trace acid can be removed by filtering through basic $\mathrm{Al}_{2} \mathrm{O}_{3}$ ). Enantiomerically pure diaza-cisdecalin was prepared as previously described. ${ }^{1}$ The $\mathrm{Cu}(\mathrm{TMEDA}) \mathrm{Cl}(\mathrm{OH})$ catalyst was prepared $^{2}$ and used in the oxidative biaryl coupling reactions to prepare the racemic samples of the biaryl products.

Analytical thin layer chromatography (TLC) was performed on EM Reagents 0.25 $\mathrm{mm}$ silica-gel 60-F plates. Visualization was accomplished with UV light. Chromatography was performed using a forced flow of the indicated solvent system on EM Reagents Silica Gel 60 (230-400 mesh). ${ }^{3}$ Enantiomeric excesses were determined using analytical high performance liquid chromatography (HPLC), performed on a Waters 600 HPLC with UV detection at $254 \mathrm{~nm}$. An analytical Chiralpak AD column $(0.46 \mathrm{~cm} \times 25 \mathrm{~cm})$ from Daicel was used. ${ }^{1} \mathrm{H}$ NMR spectra were recorded on Bruker AM-500 (500 MHz), AM-360 (360 MHz), AM-250 (250 MHz), or AM-200 (200 MHz) spectrometers. Chemical shifts are reported in ppm from tetramethylsilane $(0 \mathrm{ppm})$ or from the solvent resonance $\left(\mathrm{CDCl}_{3} 7.26 \mathrm{ppm}\right)$. Data are reported as follows: chemical shift, multiplicity $(\mathrm{s}=$ singlet, $\mathrm{d}=$ doublet, $\mathrm{t}=$ triplet, $\mathrm{q}=$ quartet, $\mathrm{br}=$ broad, $\mathrm{m}=$ 
multiplet), coupling constants, and number of protons. Decoupled ${ }^{13} \mathrm{C}$ NMR spectra were recorded on a Bruker AM-500 (125 MHz) spectrometer. IR spectra were taken on a Perkin-Elmer FT-IR spectrometer using a thin film on $\mathrm{NaCl}$ plates or a $\mathrm{CHCl}_{3}$ solution. Melting points were obtained on Thomas Scientific Unimelt apparatus and are uncorrected. Optical rotations were measured on a Perkin-Elmer Polarimeter 341 with a sodium lamp and are reported as follows $[\alpha]_{\lambda}^{\mathrm{T}},(\mathrm{c} \mathrm{g} / 100 \mathrm{~mL}$, solvent).<smiles>COc1cc(CC(=O)C(C(C)=O)C(C)=O)cc(OC)c1</smiles>

Methyl-1,3-dihydroxy-6,8-dimethoxynapthalene-2-carboxylate (6). To a solution of phenyl acetic acid $5(0.429 \mathrm{~g}, 2.19 \mathrm{mmol})$ in $\mathrm{CH}_{2} \mathrm{Cl}_{2}, \mathrm{SOCl}_{2}(0.319 \mathrm{~mL})$ was added. After $1 \mathrm{~h}$ at reflux, the solution was concentrated. To a suspension of $\mathrm{NaH}(0.156$ $\mathrm{g}, 6.5 \mathrm{mmol})$ in THF $(51 \mathrm{~mL})$ was added dimethyl malonate $(0.838 \mathrm{~g}, 6.35 \mathrm{mmol})$. After stirring for $1 \mathrm{~h}$ a solution of the unpurified acid chloride in THF was added. After $1 \mathrm{~h}$ at room temperature, $1 \mathrm{M} \mathrm{HCl}$ and EtOAc were added. The layers were separated and the aqueous layer was extracted three times with EtOAc. The organic layers were combined, washed with brine, dried over $\mathrm{MgSO}_{4}$, filtered, and concentrated. The residue was chromatographed $\left(\mathrm{SiO}_{2} ; 85 \%\right.$ hexanes/EtOAc) to afford the intermediate tricarbonyl $(0.650 \mathrm{~g})$ as a clear oil.<smiles>COC(=O)c1c(O)cc2cc(OC)cc(OC)c2c1OC(C)=O</smiles> 


\section{Methyl-1-acetoxy-3-hydroxy-6,8-dimethoxynapthalene-2-carboxylate (7). To}

a solution of the intermediate tricarbonyl in methane sulfonic acid $(10 \mathrm{~mL})$, was added $\mathrm{P}_{2} \mathrm{O}_{5}(0.600 \mathrm{~g})$. After $3 \mathrm{~h}$ at room temperature, ice was added and the resulting precipitate was filtered and dried in an oven overnight to yield naphthalene diol $(0.500 \mathrm{~g})$ in $82 \%$ yield (from the phenylacetic acid 5) as a grey solid: $\mathrm{mp} 162-164{ }^{\circ} \mathrm{C} ;{ }^{1} \mathrm{H} \mathrm{NMR}$ $\left.\left(500 \mathrm{MHz}, \mathrm{CDCl}_{3}\right) \delta\right), 11.13(\mathrm{~s}, 1 \mathrm{H}), 10.70(\mathrm{~s}, 1 \mathrm{H}), 6.64(\mathrm{~s}, 1 \mathrm{H}), 6.48(\mathrm{~d}, J=2.2 \mathrm{~Hz}, 1 \mathrm{H})$, $6.28(\mathrm{~d}, J=2.2 \mathrm{~Hz}, 1 \mathrm{H}), 4.05(\mathrm{~s}, 3 \mathrm{H}), 4.02(\mathrm{~s}, 3 \mathrm{H}), 3.89(\mathrm{~s}, 3 \mathrm{H}) ;{ }^{13} \mathrm{C} \mathrm{NMR}(125 \mathrm{MHz}$, $\left.\mathrm{CDCl}_{3}\right) \delta 171.3,160.7,158.8,157.7,141.1,105.1,102.0,97.9,97.4,95.8,56.1,55.3$, 52.4; IR (film) 3443, 3304, 2953, 1664, $1594 \mathrm{~cm}^{-1}$; HRMS (ES) calcd for $\mathrm{C}_{14} \mathrm{H}_{14} \mathrm{O}_{6}$ $\left(\mathrm{MH}^{+}\right)$279.0868, found 279.0880. This sequence matches that of another synthesis but no experimental procedures or characterizations were reported. ${ }^{4}$

To a round bottom flask containing the naphthalene diol $(0.50 \mathrm{~g}, 1.80 \mathrm{mmol})$, $\mathrm{Ac}_{2} \mathrm{O}(2.54 \mathrm{~mL})$ and pyridine $(2.50 \mathrm{~mL})$ were added. After $4 \mathrm{~h}$ at room temperature, The solution was poured over ice to quench any excess $\mathrm{Ac}_{2} \mathrm{O}$. EtOAc was added and the organic layer was subsequently washed with $1 \mathrm{M} \mathrm{HCl}$, water, brine, dried over $\mathrm{MgSO}_{4}$, filtered, and concentrated. Methanol was added to this diacetate material along with sufficient $1 \mathrm{M} \mathrm{NaOMe}$ solution until TLC analysis indicated no remaining diacetate. $1 \mathrm{M}$ $\mathrm{HCl}$ was subsequently added and the aqueous layer was extracted three times with $\mathrm{CH}_{2} \mathrm{Cl}_{2}$. The combined organic extracts were dried over $\mathrm{MgSO}_{4}$, filtered, and concentrated. The residue was chromatographed $\left(\mathrm{SiO}_{2} ; 70 \%\right.$ hexanes/EtOAc) to afford naphthol 7 (0.44 g) in 76\% yield as a yellow solid: $\mathrm{mp} 168-169{ }^{\circ} \mathrm{C} ;{ }^{1} \mathrm{H}$ NMR $(500 \mathrm{MHz}$, $\left.\mathrm{CDCl}_{3}\right) \delta 10.83(\mathrm{~s}, 1 \mathrm{H}), 7.06(\mathrm{~s}, 1 \mathrm{H}), 6.52(\mathrm{~d}, J=2.2 \mathrm{~Hz}, 1 \mathrm{H}), 6.29(\mathrm{~d}, J=2.2 \mathrm{~Hz}, 1 \mathrm{H})$, $3.98(\mathrm{~s}, 3 \mathrm{H}) 3.89(\mathrm{~s}, 3 \mathrm{H}), 3.88(\mathrm{~s}, 3 \mathrm{H}), 2.37(\mathrm{~s}, 3 \mathrm{H}) ;{ }^{13} \mathrm{C} \mathrm{NMR}\left(125 \mathrm{MHz}, \mathrm{CDCl}_{3}\right) \delta$ 
$170.0,169.5,161.1,158.1,157.4,149.9,141.1,111.2,109.3,107.3,97.8,97.1,56.1$, 55.4, 52.9, 20.9; IR (film) 3134, 3007, 2957, 2845, 1760, 1671, 1629, $1571 \mathrm{~cm}^{-1}$; HRMS (ES) calcd for $\mathrm{C}_{16} \mathrm{H}_{16} \mathrm{O}_{7} \mathrm{Na}\left(\mathrm{MNa}^{+}\right)$343.0794, found $\left(\mathrm{MNa}^{+}\right) 343.0797$.

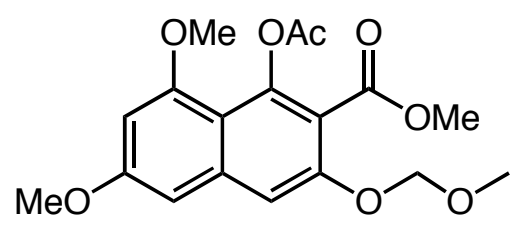

\section{Methyl-1-acetoxy-6,8-dimethoxy-3-(methoxymethoxy)naphthalene-2-}

carboxylate (8). A solution of $7(0.250 \mathrm{~g}, 0.780 \mathrm{mmol})$ in $\mathrm{DMF}(4 \mathrm{~mL})$ was cooled to 0 ${ }^{\circ} \mathrm{C}$ at which time $\mathrm{NaH}(0.028 \mathrm{~g}, 1.17 \mathrm{mmol})$ was added. After $20 \mathrm{~min}, \mathrm{MOMCl}(0.099$ $\mathrm{mL}$ ) was added in one portion. After $2 \mathrm{~h}$ at room temperature, EtOAc and $1 \mathrm{M} \mathrm{HCl}$ were added. The organic layer was washed three times with water and brine to remove DMF. The organic layer was dried over $\mathrm{MgSO}_{4}$, filtered, and concentrated. The residue was chromatographed $\left(\mathrm{SiO}_{2} ; 70 \%\right.$ hexanes/EtOAc) to afford $8(0.270 \mathrm{~g})$ in $94 \%$ yield as a white powder: mp $140-141{ }^{\circ} \mathrm{C} ;{ }^{1} \mathrm{H}$ NMR $\left(500 \mathrm{MHz}, \mathrm{CDCl}_{3}\right) \delta 7.22(\mathrm{~s}, 1 \mathrm{H}), 6.65(\mathrm{~d}, J=$ $2.2 \mathrm{~Hz}, 1 \mathrm{H}), 6.39$ (d, $J=2.2 \mathrm{~Hz}, 1 \mathrm{H}), 5.28$ (s, 2H), $3.92(\mathrm{~s}, 3 \mathrm{H}), 3.87$ (s, 3H), 3.86 (s, 3H), $3.51(\mathrm{~s}, 3 \mathrm{H}), 2.31(\mathrm{~s}, 3 \mathrm{H}) ;{ }^{13} \mathrm{C}$ NMR $\left(125 \mathrm{MHz}, \mathrm{CDCl}_{3}\right) \delta$ 169.3, 165.8, 159.9, $157.3,152.5,145.6,138.8,116.8,110.4,107.9,98.8,98.6,95.1,56.6,56.3,55.6,52.7$, 20.9; IR (film) 3067, 2989, 1772, 1714, 1629, $1586 \mathrm{~cm}^{-1}$; HRMS (ES) calcd for $\mathrm{C}_{18} \mathrm{H}_{20} \mathrm{O}_{8} \mathrm{Na}\left(\mathrm{MNa}^{+}\right)$364.1158, found 364.1164.<smiles>COCOc1cc2cc(OC)cc(OC)c2c(O)c1C(=O)CS(C)=O</smiles>

1,3-dimethoxy-6-(methoxymethoxy)-7-(2-(methylsulfinyl)acetyl)naphthalene-

8-yl-acetate (9). Benzene $(10 \mathrm{~mL})$ and DMSO $(3.1 \mathrm{~mL})$ were added to a round bottom 
flask containing $\mathrm{NaH}(0.296 \mathrm{~g}, 12.33 \mathrm{mmol})$. After $1 \mathrm{~h}$ at reflux, the reaction mixture was allowed to cool to room temperature and a solution of $\mathbf{8}(0.642 \mathrm{~g}, 1.76 \mathrm{mmol})$ in benzene was added. After $1 \mathrm{~h}$ at $45^{\circ} \mathrm{C}$, the solution was concentrated at which time water and acetic acid were added dropwise until a precipitate formed. The precipitate was filtered and dried to afford $9(0.600 \mathrm{~g})$ in $96 \%$ yield as a yellow powder: mp 145-146 ${ }^{\circ} \mathrm{C} ;{ }^{1} \mathrm{H}$ NMR (500 MHz, $\mathrm{CDCl}_{3}$ ) $\delta 14.05$ (s, 1H), 6.73 (s, 1H), 6.54 (d, J=2.2 Hz, 1H), $6.36(\mathrm{~d}, J=2.2 \mathrm{~Hz}, 1 \mathrm{H}), 5.35(\mathrm{dd}, J=9.7, J=6.8,2 \mathrm{H}), 4.84(\mathrm{~d}, J=14 \mathrm{~Hz}, 1 \mathrm{H}), 4.30(\mathrm{~d}$, $J=14 \mathrm{~Hz}, 1 \mathrm{H}), 3.98(\mathrm{~s}, 3 \mathrm{H}), 3.89(\mathrm{~s}, 3 \mathrm{H}), 3.56(\mathrm{~s}, 3 \mathrm{H}), 2.77(\mathrm{~s}, 3 \mathrm{H}) ;{ }^{13} \mathrm{C}$ NMR $(125$ $\left.\mathrm{MHz}, \mathrm{CDCl}_{3}\right) \delta 194.6,165.3,162.3,160.7,154.0,141.6,108.1,107.3,100.2,99.1,97.2$, 94.8, 68.6, 56.8, 56.2, 55.4, 39.9; IR (film) 3003, 2968, 3397, 1625, $1583 \mathrm{~cm}^{-1}$; HRMS (ES) calcd for $\mathrm{C}_{17} \mathrm{H}_{20} \mathrm{O}_{7} \mathrm{SNa}\left(\mathrm{MNa}^{+}\right)$368.0930, found 368.0937.

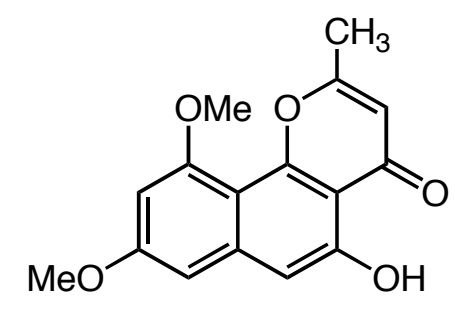

Flavasperone (4). To a solution of $9(0.192 \mathrm{~g}, 0.523 \mathrm{mmol})$ in toluene $(10 \mathrm{~mL})$, one drop of piperdine was added. The solution was warmed to $45{ }^{\circ} \mathrm{C}$ and newly purchased acetaldehyde $(0.375 \mathrm{~mL})$ was added. After $3 \mathrm{~h}$ at reflux, the mixture was allowed to cool to room temperature upon which time EtOAc and $1 \mathrm{M} \mathrm{HCl}$ were added. The organic layer was washed with water and brine. To the unpurified product was added $\mathrm{CH}_{2} \mathrm{Cl}_{2}(3.80 \mathrm{~mL})$ and the reaction mixture was cooled to $-78{ }^{\circ} \mathrm{C}$. The cooled solution was treated with $\mathrm{BCl}_{3}(0.476 \mathrm{~mL}, 3.00 \mathrm{mmol})$ and was allowed to stir for 20 minutes at which time $\mathrm{NaHCO}_{3}$ was added. The reaction mixture was then allowed to warm to room temperature. The organic layer was washed with water and brine. The 
residue was chromatographed $\left(\mathrm{SiO}_{2} ; 4 \% \mathrm{EtOAc} / \mathrm{CH}_{2} \mathrm{Cl}_{2}\right)$ to afford $4(0.036 \mathrm{~g})$ in $65 \%$ yield as a yellow solid: $\left(500 \mathrm{MHz}, \mathrm{CDCl}_{3}\right) \delta 12.83(\mathrm{~s}, 1 \mathrm{H}), 6.89(\mathrm{~s}, 1 \mathrm{H}), 6.60(\mathrm{~d}, J=2.2$ Hz, 1H), 6.41 (d, J=2.2 Hz, 1H), 6.29 (s, 1H), 3.98 (s, 3H), 3.93 (s, 3H), 2.51 (s, 3H); ${ }^{13} \mathrm{C}$ NMR $\left(125 \mathrm{MHz}, \mathrm{CDCl}_{3}\right) \delta 182.9,166.4,161.7,159.6,155.9,154.7,140.6,110.6$, 110.4, 108.6, 105.2, 97.0, 96.8, 56.0, 55.2, 20.5. ${ }^{1} \mathrm{H}$ NMR matches that of reported compound. $^{5}$

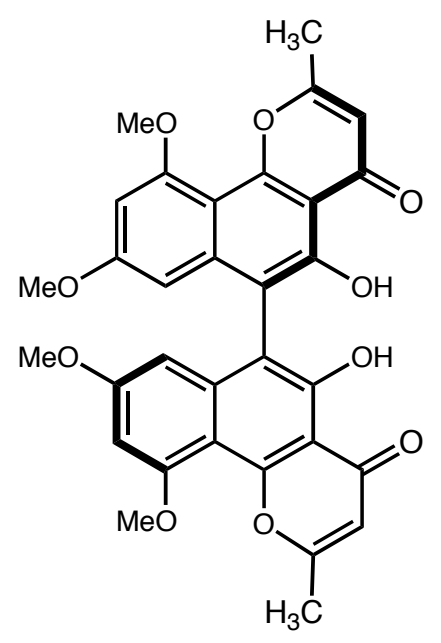

Bisisonigerone (3). To a solution of $4(0.030 \mathrm{~g}, 0.010 \mathrm{mmol})$ in $1: 1$ $\mathrm{CH}_{3} \mathrm{CN} / \mathrm{CH}_{2} \mathrm{Cl}_{2}(3 \mathrm{~mL}), \mathrm{CuI}(\mathrm{S}, S)$-1,5-diaza-cis decalin catalyst $(0.036 \mathrm{~g}, 0.010 \mathrm{mmol})$ was added. After $6 \mathrm{~d}$ at $40{ }^{\circ} \mathrm{C}, 1 \mathrm{M} \mathrm{HCl}$ was added and the aqueous layer was extracted three times with $\mathrm{CH}_{2} \mathrm{Cl}_{2}$. The combined organic extracts were dried over $\mathrm{MgSO}_{4}$, filtered, and concentrated. The residue was chromatographed $\left(\mathrm{SiO}_{2} ; 60 \%\right.$ hexanes/EtOAc) to afford $3(0.018 \mathrm{~g})$ in $60 \%$ yield along with $4(0.009 \mathrm{~g}, 80 \% \mathrm{ee})$ as a yellow solid: $\mathrm{mp} 180-185^{\circ} \mathrm{C}$ decomposition; $[\alpha]_{\mathrm{D}}^{23} \quad+72.58$ ( $0.25,80 \%$ ee, $\left.\mathrm{CHCl}_{3}\right) ;{ }^{1} \mathrm{H}$ NMR (500 MHz, CDCl $) \delta 13.19(\mathrm{~s}, 1 \mathrm{H}), 6.45(\mathrm{~d}, J=2.2 \mathrm{~Hz}, 1 \mathrm{H}), 6.33(\mathrm{~s}, 1 \mathrm{H}), 6.21$ (d, $J=2.2 \mathrm{~Hz}, 1 \mathrm{H}), 4.02(\mathrm{~s}, 3 \mathrm{H}), 3.55(\mathrm{~s}, 3 \mathrm{H}), 2.56(\mathrm{~s}, 3 \mathrm{H}) ;{ }^{13} \mathrm{C}$ NMR $(125 \mathrm{MHz}$, $\left.\mathrm{CDCl}_{3}\right) \delta 182.9,166.4,161.7,159.7,156.1,154.6,140.5,110.5,110.3,108.6,104.3$, 
96.9, 96.7, 56.0, 55.2, 29.7, 20.5; IR (film) 3366, 3003, 2926, 1656, 1610, 1579, 1517 $\mathrm{cm}^{-1}$; HRMS (ES) calcd for $\mathrm{C}_{32} \mathrm{H}_{26} \mathrm{O}_{10} \mathrm{Na}\left(\mathrm{MNa}^{+}\right)$571.1604, found 571.1624; CSP HPLC (Chiralpak AD, $1.0 \mathrm{~mL} / \mathrm{min}, 90: 10$ hexanes: $i-\operatorname{PrOH}): \mathrm{t}_{\mathrm{R}}(S)=40.6 \mathrm{~min}, \mathrm{t}_{\mathrm{R}}(R)=109.9 \mathrm{~min}$.

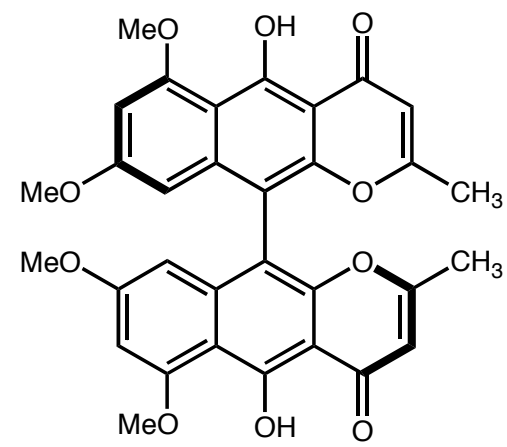

Nigerone (1). A solution of $3(0.026 \mathrm{~g}, 80 \%$ ee) in $\mathrm{MeOH}(22 \mathrm{~mL})$ was heated with sat. aq. $\mathrm{NaOH}$ solution $(0.22 \mathrm{~mL})$ and was placed in a $70^{\circ} \mathrm{C}$ oil bath. After $18 \mathrm{~h}$, $1 \mathrm{M} \mathrm{HCl}$ was added and the aqueous layer was extracted three times with $\mathrm{CH}_{2} \mathrm{Cl}_{2}$. The organic layers were combined, dried over $\mathrm{MgSO}_{4}$, filtered, and concentrated. The residue was chromatographed $\left(\mathrm{SiO}_{2} ; 60 \%\right.$ hexanes/EtOAc) to afford $\mathbf{1}(0.013 \mathrm{~g}, 77 \%$ ee) in $50 \%$ yield as yellow solid: $\mathrm{mp}>200{ }^{\circ} \mathrm{C}$ decomposition; ${ }^{1} \mathrm{H}$ NMR $\left(500 \mathrm{MHz}, \mathrm{CDCl}_{3}\right) \delta 15.31$ (s, 1H), $6.44(\mathrm{~d}, J=7 \mathrm{~Hz}, 2 \mathrm{H}), 6.07$ (d, $J=7 \mathrm{~Hz}, 1 \mathrm{H}), 6.00(\mathrm{~s}, 1 \mathrm{H}), 4.06(\mathrm{~s}, 3 \mathrm{H}), 3.49$ (s, 3H), 2.03 (s, 3H); ${ }^{13} \mathrm{C}$ NMR (125 MHz, $\left.\mathrm{CDCl}_{3}\right) \delta$ 184.5, 167.6, 163.1, 161.9, 161.2, 151.3, 140.7, 108.8, 107.3 105.5, 104.3, 97.2, 96.5, 56.2, 55.2, 20.6; IR (film) 3377, 2930, 2853, 1652, 1610, 1586, 1409; HRMS (ES) calcd for $\mathrm{C}_{32} \mathrm{H}_{26} \mathrm{O}_{10} \mathrm{Na}\left(\mathrm{MNa}^{+}\right)$ 571.1604, found 571.1624; CSP HPLC (Chiralpak AD, $1.0 \mathrm{~mL} / \mathrm{min}, 90: 10$ hexanes:i$\operatorname{PrOH}): \mathrm{t}_{\mathrm{R}}(S)=31.7 \mathrm{~min}, \mathrm{t}_{\mathrm{R}}(R)=38.7 \mathrm{~min}$.

Trituration of this material with EtOAc provided 1 with $90 \% e e:[\alpha]_{\mathrm{D}}^{23}:-223(c$ $0.018 \mathrm{~g} / 100 \mathrm{~mL}, \mathrm{CH}_{2} \mathrm{Cl}_{2}, 90 \%$ ee), literature (ref 3a, main text) $[\alpha]_{\mathrm{D}}{ }^{20}:-287.7$ (c 1.00, $\left.\mathrm{CHCl}_{3}\right)$. 


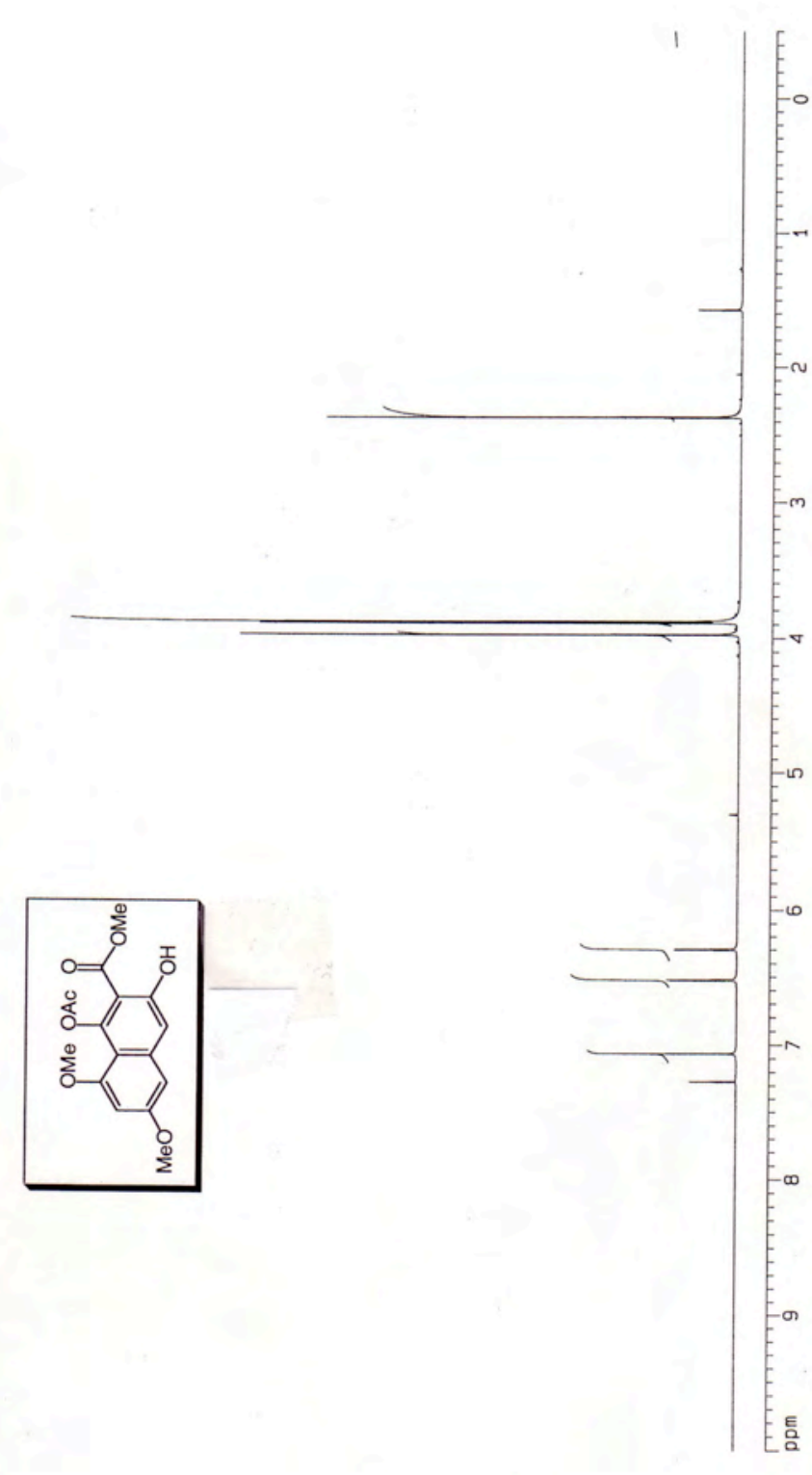




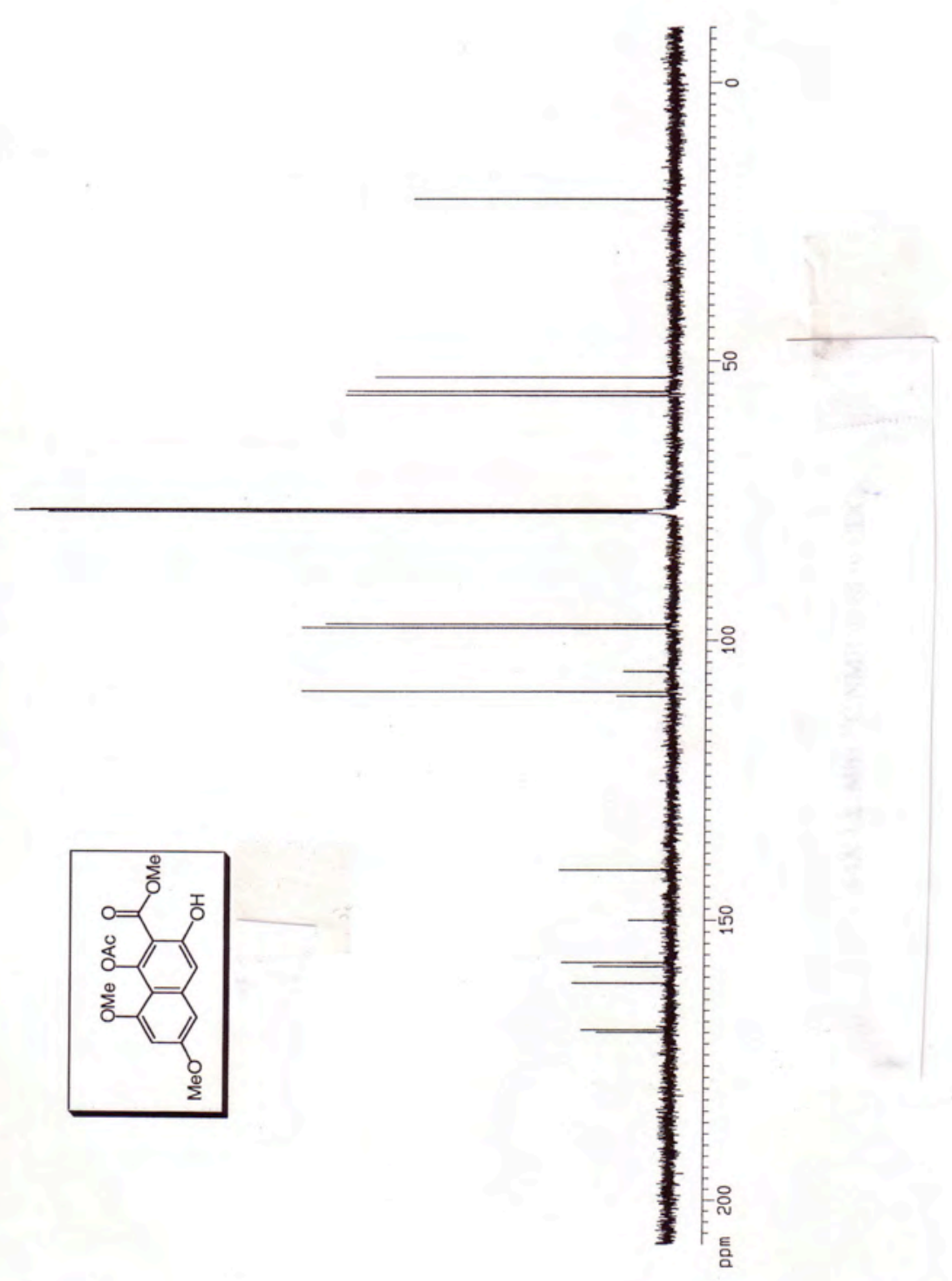

S10 


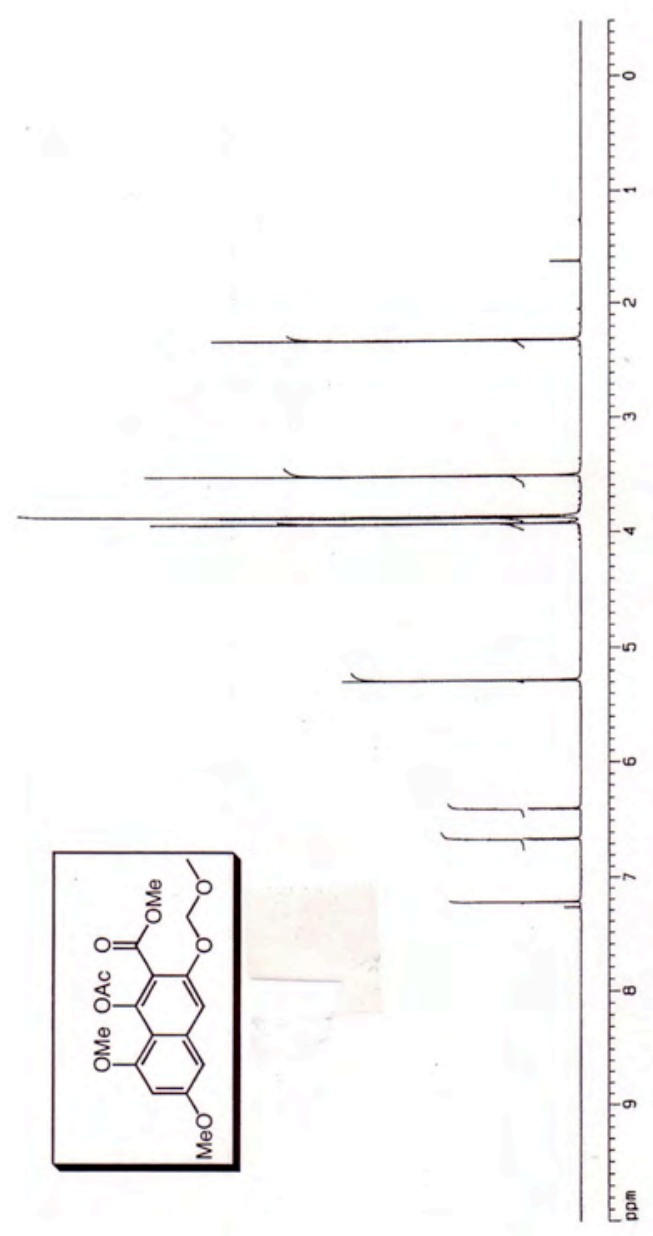




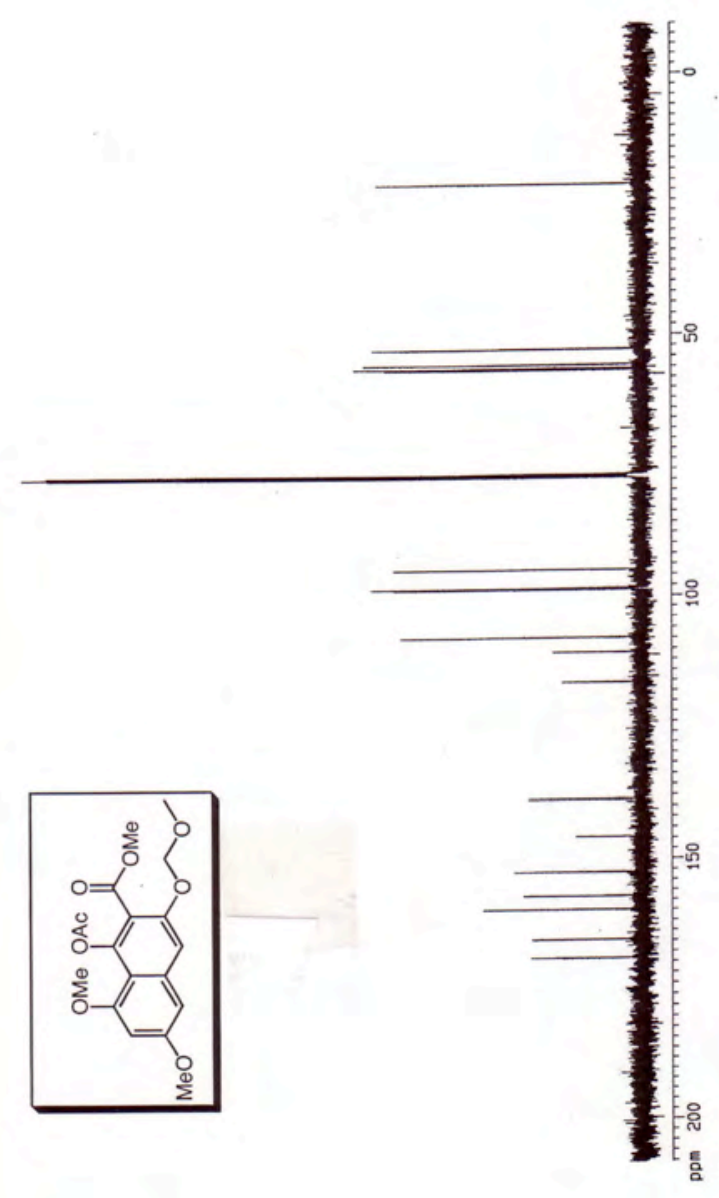

S12 


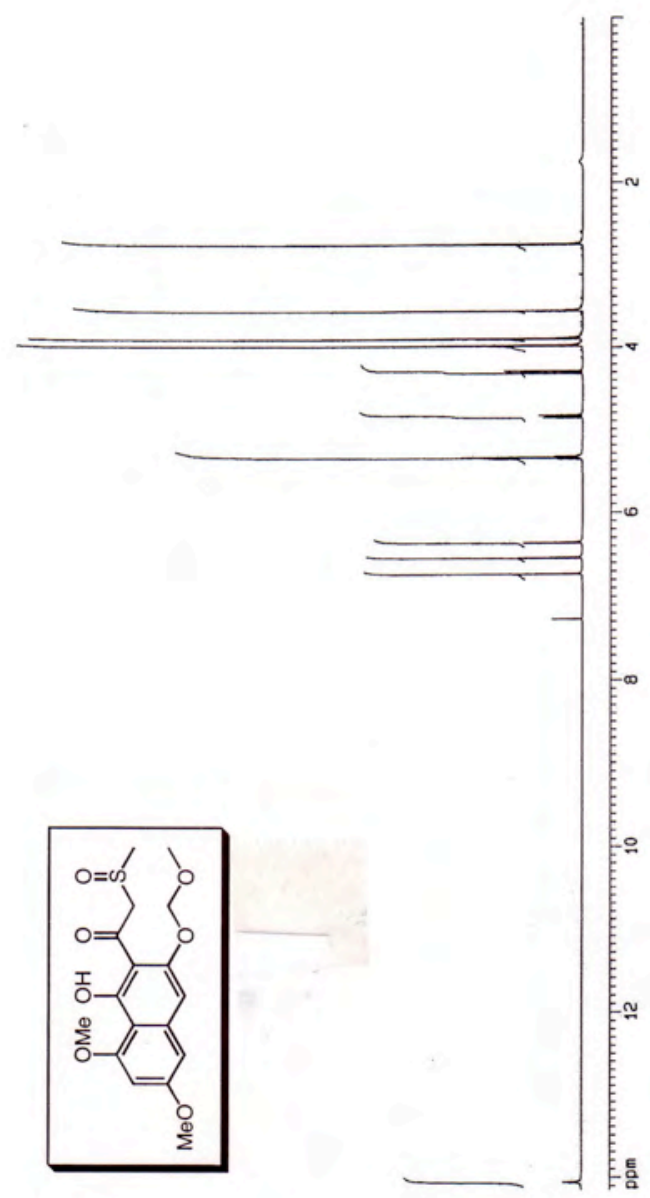

S13 


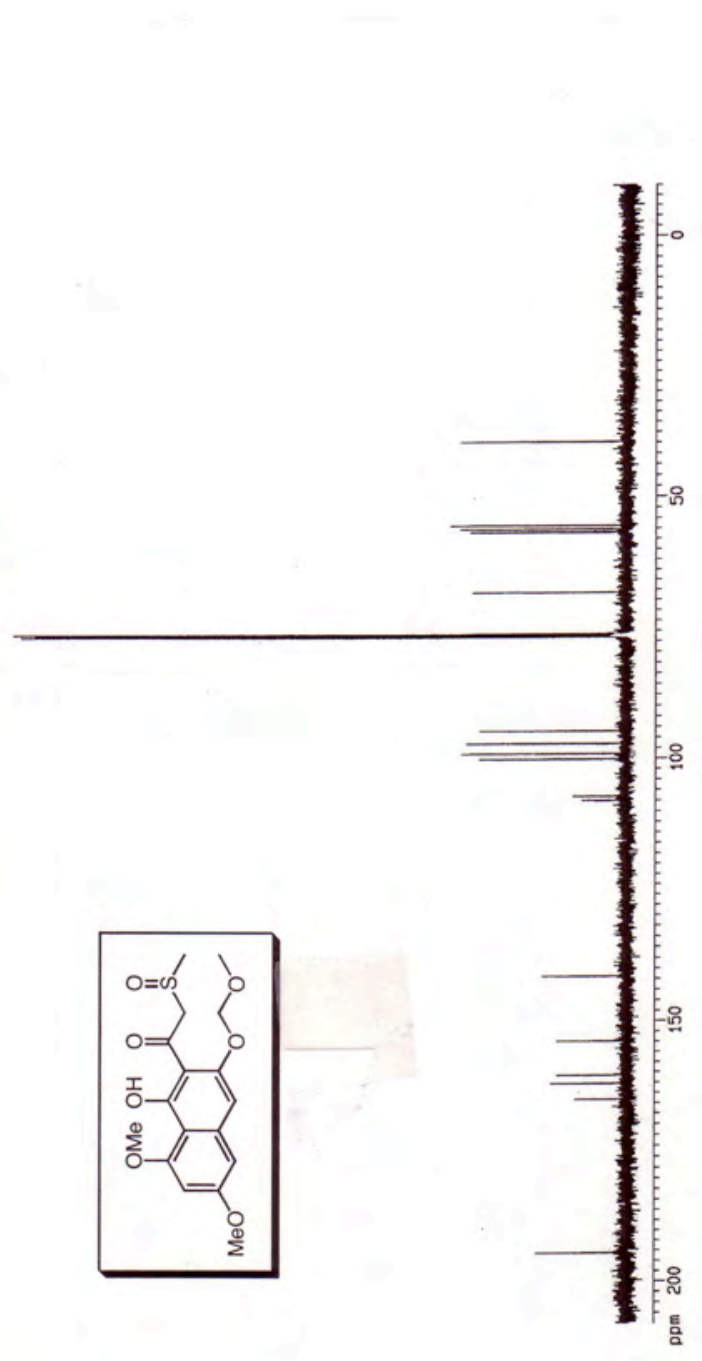




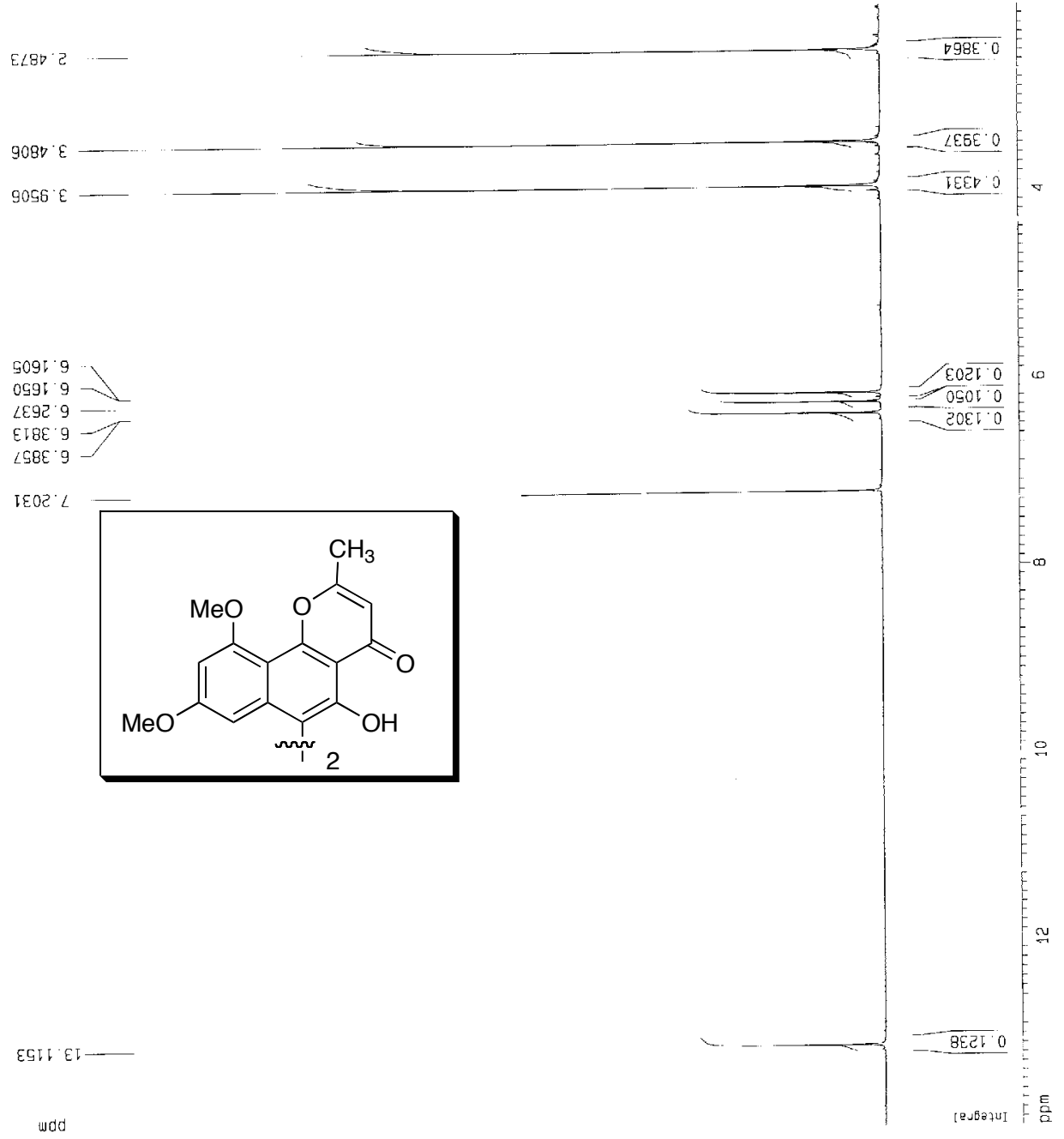

wdd

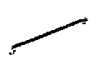



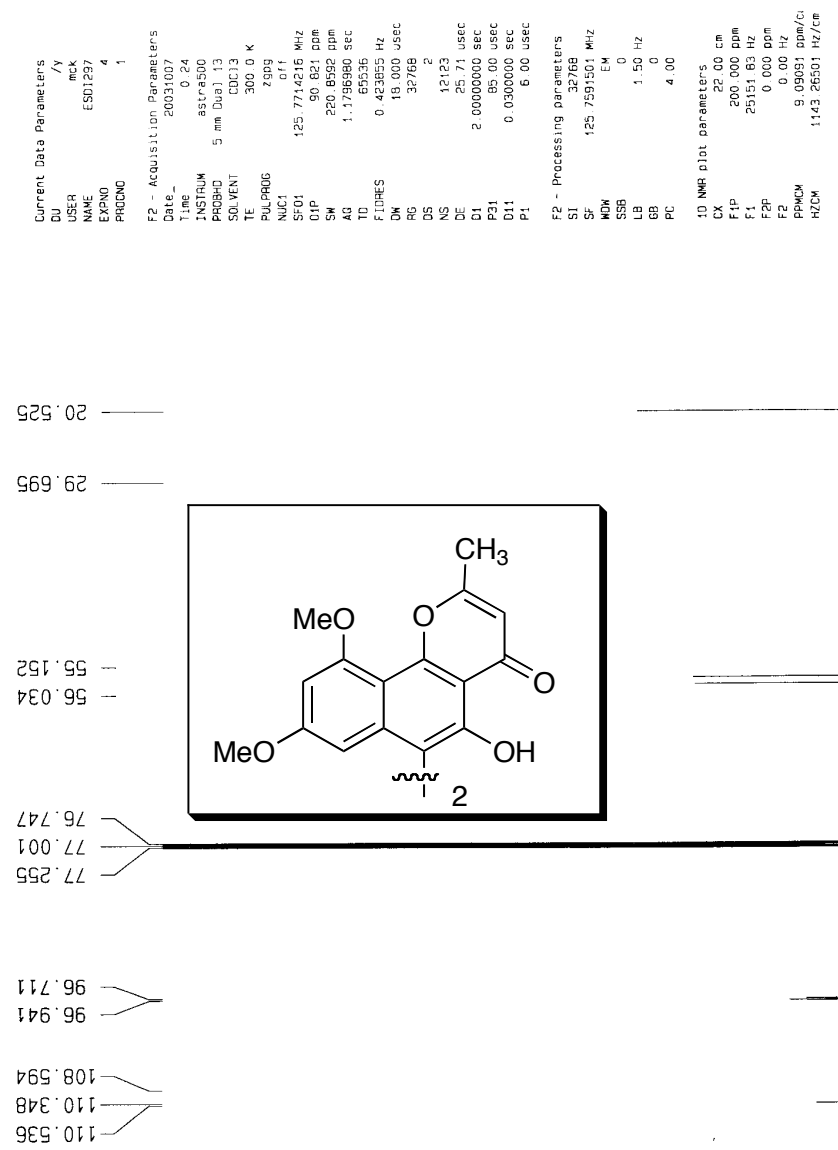

BES ODI -
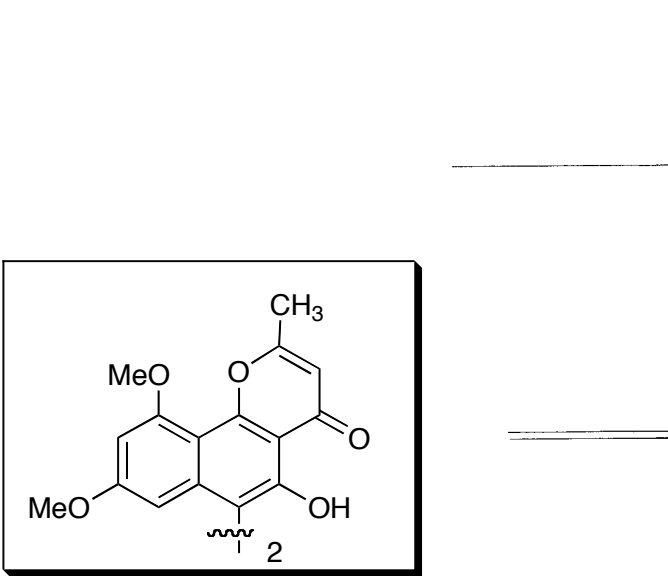

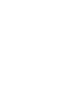




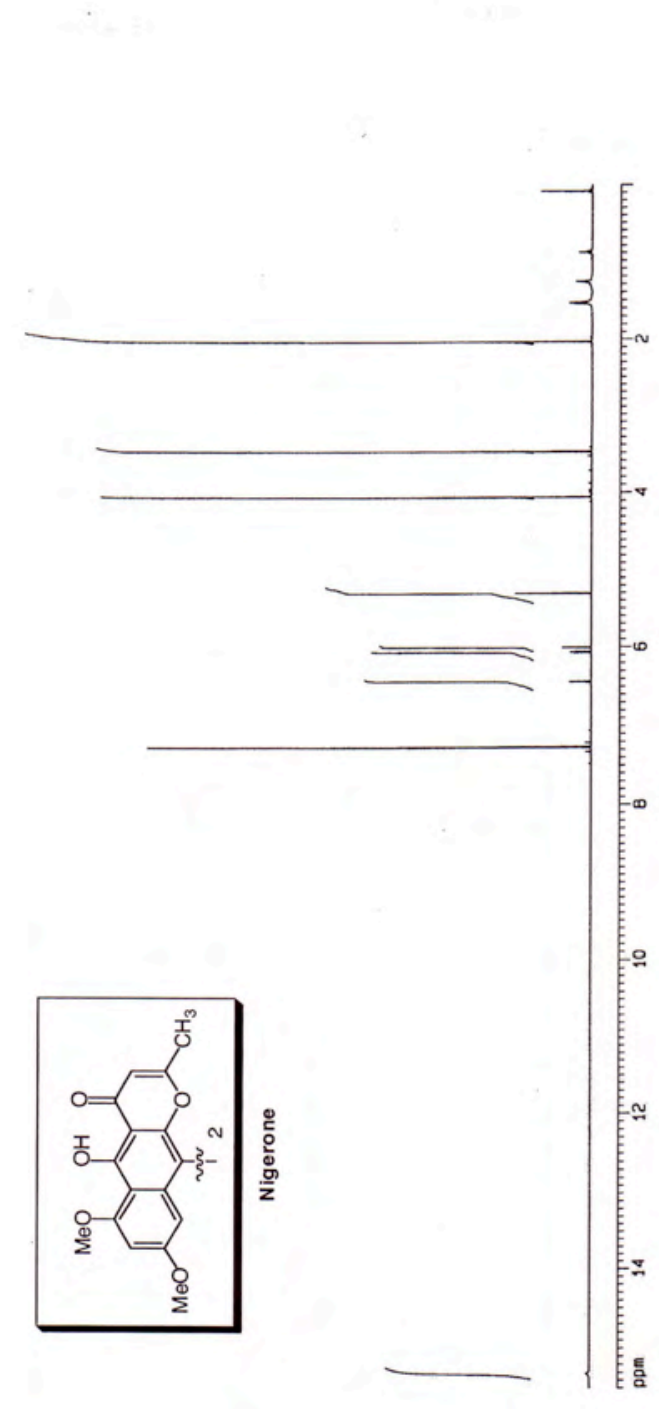




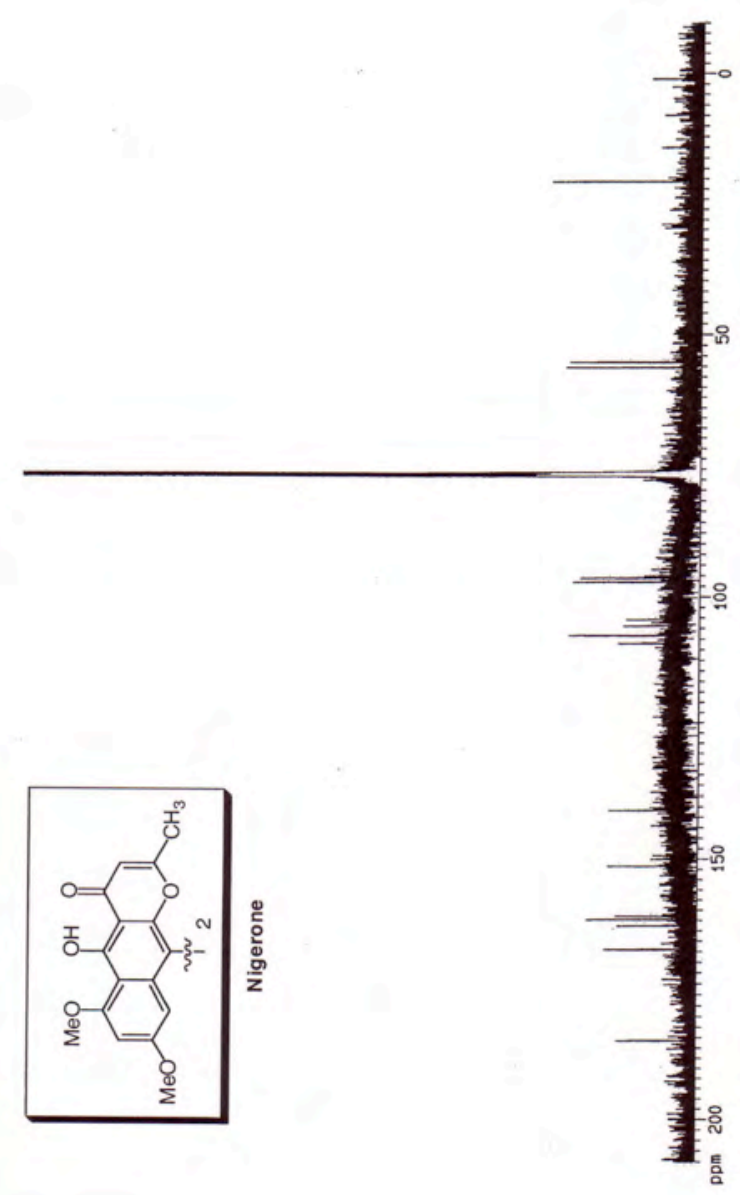


(1) Li, X.; Schenkel, L. B.; Kozlowski, M. C. Org. Lett. 2000, 2, 975-978.

(2) Nakajima, M.; Miyoshi, I.; Kanayama, K.; Hashimoto, S.-I.; Noji, M.; Koga, K. J. Org. Chem. 1999, 64, 2264-2271.

(3) Still, W. C.; Kahn, M.; Mitra, A. J. Org. Chem. 1978, 43, 2923-2925.

(4) Piettre, A.; Chevenier, E.; Massardier, C.; Gimbert, Y.; Greene, A. E. Org. Lett 2002, 4, 3139-3142.

(5) Bycroft, B.W.; Roberts, J. C. J. Chem. Soc. 1963, 4868-4872. 